\title{
Method for Improvement of Ocean Wind Speed Estimation Accuracy by Taking into Account the Relation between Wind Speed and Wind Direction
}

\author{
Kohei Arai ${ }^{1}$, Kenta Azuma ${ }^{2}$ \\ Science and Engineering Faculty, Saga University, Saga City, Japan ${ }^{1}$ \\ Former Student, Saga University, Saga City, Japan ${ }^{2}$
}

\begin{abstract}
A method for improvement of ocean wind speed estimation accuracy by taking into account the relation between wind speed and wind direction is proposed. Brightness temperature observed with microwave radiometer onboard satellite is modified with microwave radiometer derived wind direction proposed by Frank Wentz. Using the modified brightness temperature, more precise wind speed is estimated. Experiments with AMSR-E and NCEP GDAS data show improvements of wind speed estimation in comparison to the existing method based on the geophysical model of Frank Wentz together with the retrieval algorithm of Akira Shibata.
\end{abstract}

Keywords-Sea surface wind speed (WS); advanced microwave scanning radiometer for erath observing system (AMSR-E); NCEP Global Data Assimilation System (GDAS); relative wind direction (RWD)

\section{INTRODUCTION}

The physical quantity can be estimated. This is called remote sensing. Remote sensing generally has visible, nearinfrared, thermal-infrared, and microwave observation wavelengths, and sensors are used [1]. Compared to other wavelengths, remote sensing that measures microwaves has poor resolution and can only make rough measurements [2]. However, it has the advantage of being observable even if it is affected by clouds, regardless of day or night. Furthermore, at present, the wind speed on the ocean surface can be remotely sensed only by sensors in the microwave wavelength range [3].

However, it is known that the estimation accuracy of the wind speed changes due to the influence of the relative wind direction in the marine physical quantity cage determination using microwaves. Frank. Wentz (2002) [4] describes the microwave radiation transfer equation and the marine physical quantity using the microwave radiation transfer equation when using the Top of Atmosphere (TOA) obtained from the microwave radiometer AMSR. We created an equation to estimate. Observation of atmosphere, ocean, and land with multi-wavelength microwave radiometer is introduced [5]. At this time, the influence of the relative wind direction was also taken into consideration. However, this effect was used in the microwave radiometer SSM / I and cannot be expected in AMSR. This is because AMSR and ASMR / I have different observation frequency bands.
Arai and Sakakibara proposed a method using the Wentz algorithm using improved simulated annealing for the radiometer ASMR-E [6]. This made it possible to simultaneously estimate various marine physical quantities, which led to an improvement in the estimation structure. On the other hand, Konda, Shibata, Ebuchi, and Arai (2006) used the estimated wind speed obtained from the AMSR product mounted on the observation satellite ADEOS-II and the wind direction obtained from the microwave scatterometer, SeaWinds onboard on the satellite [7].

One of the important issues of the improvement of ocean wind speed estimation accuracy is how to take into account the influence due to wind direction dependency from the wind speed estimation. This is the issue of this paper. The influence of the relative wind direction on the wind speed estimation was investigated, and the index of the influence of the relative wind direction under various situations was derived. They confirmed that the estimation accuracy was improved by performing the estimation using the derived index in the wind speed cage determination of ASMR and ASMR-E. However, in the case of simultaneous estimation of physical quantities, a method of modifying only the wind speed after estimation causes a contradiction in terms of estimation means. Therefore, it is desirable to influence the relative wind direction on the brightness temperature for simultaneous estimation. Therefore, in this study, the effect of relative wind direction is used using the global meteorological data (GDAS) obtained from the US National Environmental Forecast Center NCEP and the observed brightness temperature of the microwave radiometer AMSR-E mounted on the meteorological satellite aqua [8]. By analyzing the above, we analyzed how the relative wind direction affects the brightness temperature. Furthermore, by approximating the influence of the relative wind direction with several equations, we created a model that can derive the influence due to wind direction by linear calculation. By modifying Wentz's algorithm for estimating marine physical quantities with the model, the accuracy of marine wind speed estimation was improved. Experiments with AMSR-E and NCEP GDAS data show improvements of wind speed estimation in comparison to the existing method based on the geophysical model of Frank Wentz together with the retrieval algorithm of Akira Shibata. 
The following section describes related research works followed by theoretical background. Some experiments are described. After that conclusion is described together with some discussions.

\section{RELATED RESEARCH WORK}

Simplified expression of the radiative transfer equation in thermal infrared window spectrum is proposed [9]. Evaluation of vector winds observed by NSCAT in the seas around Japan is conducted [10]. Polarization sensitivity of the ocean surface together with wind vector derived from POLDER and NSCAT on ADEOS is also evaluated [11].

Estimation of SST, wind speed and water vapor with microwave radiometer data based on simulated annealing is conducted and validated with the truth data [12] together with simultaneous estimation of sea surface temperature, wind speed and water vapor with AMSR-E data based on improved simulated annealing [13].

Space and time retrieval of tide wind speed and wave height with altimeters onboard satellites based on PostGIS system is conducted [14]. Estimation of SST, wind speed and water vapor with microwave radiometer data based on simulated annealing is also conducted [15].

Correction of the effect of relative wind direction on wind speed derived by AMSR is conducted [16]. Data fusion between microwave and thermal infrared radiometer data and its application to skin sea surface temperature, wind speed and salinity retrievals are proposed [17].

Comparative study of optimization methods for estimation of Sea Surface Temperature: SST and Ocean Wind: OW with microwave radiometer data is conducted [18].

\section{TheOrETICAL BACKGROUND}

Effect of relative wind direction is considered based on the physical background. It is known that the brightness temperature of the upper atmosphere of microwaves largely depends on the gradient distribution of the sea surface and the state of the sea surface.

When the wind blows on the sea surface, the gradient distribution of the sea surface changes, and the brightness temperature at the upper end of the atmosphere fluctuates greatly. By utilizing this action, the sea wind speed can be estimated from the observed brightness temperature of a microwave radiometer or a microwave scatterometer. It is also known that the gradient distribution is distorted in the wind direction due to the wind.

It is considered that the upper-atmospheric brightness temperature changes further due to the distortion of the gradient distribution, that is, the upper-atmospheric brightness temperature also depends on the difference between the wind direction and the observation direction. The difference between the azimuth of the wind and the azimuth of the observation is called "Relative Wind Direction (RWD)". In other words, the upper atmosphere brightness temperature is considered to depend on the relative wind direction. The microwaves observed by the microwave radiometer mounted on the satellite can be roughly divided into three elements as shown in Fig. 1.

$T B_{\text {air emission }}$ is microwaves emitted by the atmosphere, and $T B_{\text {ref }}$ is microwaves emitted by the atmosphere scattered on the surface of the sea. $T B$ sea emission is a microwave emitted from the sea surface.

The sum of each is the observed brightness temperature of the radiometer, which can be expressed by Eq. (1).

$\mathrm{TB}=\mathrm{TB}_{\text {air emission }}+\mathrm{TB}_{\text {ref }}+\mathrm{TB}$ sea emission

First, consider $T$. If the sea level is a calm mirror surface, $T$ is expressed by Eq. (2).

$\mathrm{TB}_{0 \mathrm{ref}}=\mathrm{R}_{0 \mathrm{p}} \mathrm{TB}_{\text {|air }}$

$R_{0 p}$ is the reflectance, the subscript 0 is the mirror surface, and the subscript $p$ is the polarization. Also, $T B_{\mid a i r}$ means downward atmospheric radiation. $R_{0 p}$ is expressed by Eq. (3) from Fresnel's reflection law.

$R_{0 p}=\left|\rho_{p}\right|^{2}, \rho_{p}=\sqrt{\rho_{v}^{2}+\rho_{h}^{2}}$

$\rho_{v}=\frac{\varepsilon \cos \theta_{i}-\sqrt{\varepsilon-\sin ^{2} \theta_{i}}}{\varepsilon \cos \theta_{i}+\sqrt{\varepsilon-\sin ^{2} \theta_{i}}}$

$\rho_{h}=\frac{\varepsilon \cos \theta_{i}-\sqrt{\varepsilon-\sin ^{2} \theta_{i}}}{\varepsilon \cos \theta_{i}+\sqrt{\varepsilon-\sin ^{2} \theta_{i}}}$

where $\rho$ is the reflectance coefficient of Fresnel, the subscripts $v$ and $h$ are vertically polarized waves and horizontally polarized waves, and $\varepsilon$ is the complex permittivity, and $\theta_{i}$ is the angle of incidence. Also, as shown in Fig. 2, the angle of incidence and the angle of reflection are equal. Furthermore, since the emissivity of the sea surface is obtained by subtracting the reflectance from $1, T B$ sea emission can be formulated in the same manner.

The sum of these and the radiation from the atmosphere is the brightness temperature of the satellite-mounted microwave radiometer. Assuming that the observation dip of the microwave radiometer is the observation azimuth, this microwave radiometer can detect microwave radiation traveling in the direction of the zenith and azimuth. Although it is necessary to consider the beam width when actually calculating, the beam width is not considered because this chapter only explains the concept.

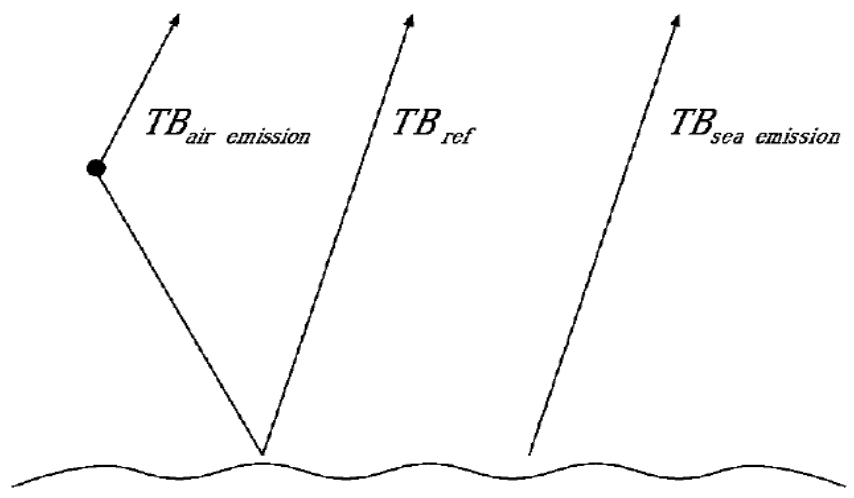

Fig. 1. Observed Brightness Temperature. 


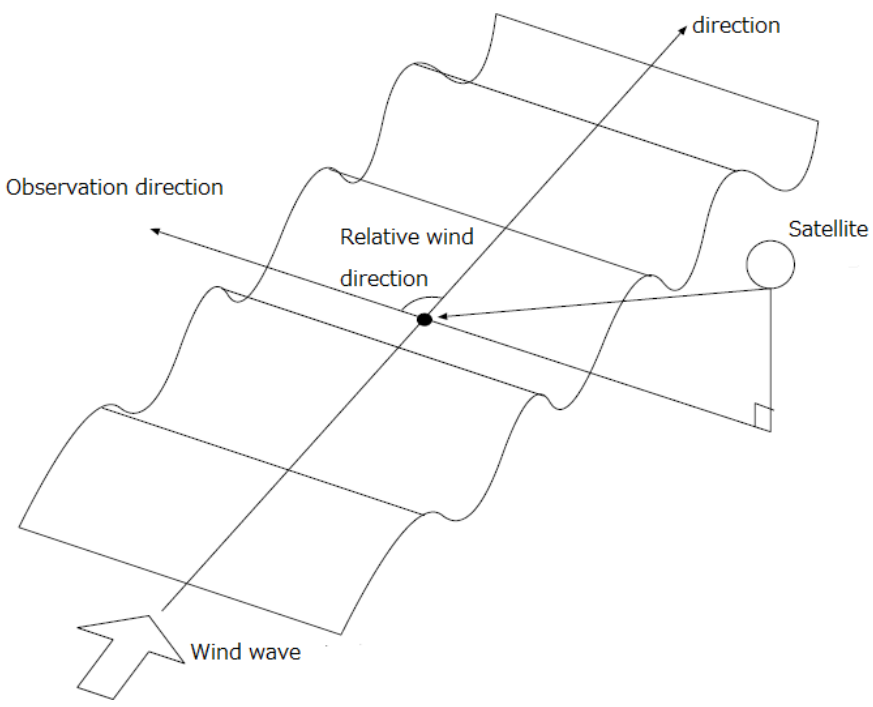

Fig. 2. Relative Wind Direction.

The observed brightness temperature $T B_{\text {ref }}$ value of the microwave radiometer is the component of the zenith angle that scatters on the sea surface at the azimuth angle. Therefore, only the components of the zenith angle scattered in the azimuth angle are considered. In this paper, the angle of incidence in this case is called the observed angle of incidence. The actual sea level creates a complex gradient distribution due to various causes such as wind waves and swells.

Due to such sea level gradients, downward atmospheric radiation is scattered in all directions. It is said that the gradient distribution of the sea surface can be approximated by the Gaussian distribution, but strictly speaking, it is considered that the gradient distribution is distorted in the wind direction by the wind. That is, the wave created by the wind becomes a wave that travels in parallel with the wind direction, and the gradient distribution is biased. Although it is difficult to know the specific degree of bias, it is easy to imagine that winds and wind waves have characteristics that depend on the observation azimuth and the angle of the wind direction, as shown in Fig. 2. That is, the variance of the gradient distribution parallel to the wind direction is large, and the variance of the gradient distribution perpendicular to the wind direction is small. Therefore, the average observer firing angle differs between the case where the observation azimuth is parallel to the wind direction and the case where the observation azimuth is perpendicular to the wind direction.

When considering the local wave gradient, it is common to divide the wave into the smallest surfaces. By doing so, the reflection of the rough sea surface can be thought of as a specular reflection as shown in Fig. 3. In this paper, the average observation angle of the observation area at this time is set to the wind direction with the azimuth angle ( $R W D=0$ deg. and / or 180 deg.), as shown in Fig. 4.

Temporarily, $\theta_{i 1}=x^{0}$, then, $\theta_{i 2}>x^{0}, \theta_{i 3}<x^{0}, \theta_{i 4}=x^{0}$. That is, $\theta_{m}=x^{0}$. In this case, there is also the influence of the rotation of the plane of polarization. Therefore, the following discussion advances to the front bank that the influence of the rotation of the plane of polarization is small. Next, when the observation azimuth is perpendicular to the wind direction (RWD = 90 deg.), the observation incident angle changes as shown in Fig. 5.

Therefore, it is considered that the average incident angle increases as the relative wind direction approaches the vertical. As can be seen from equations (3) to (5), the reflectance changes as the incident angle changes. In addition, the state of change in reflectance differs between vertically polarized waves and horizontally polarized waves. Fig. 6 shows an example of the change in reflectance when the angle of incidence changes.

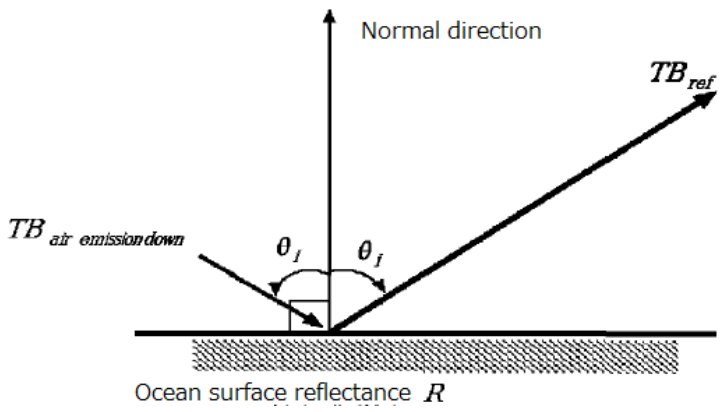

Fig. 3. Specular Reflection.

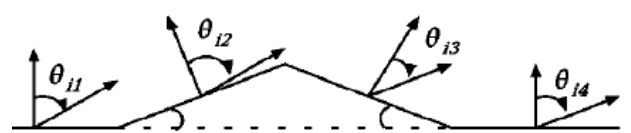

Fig. 4. Incidence Angle when Microwave Radiometer Observes in Parallel with Wind Direction.

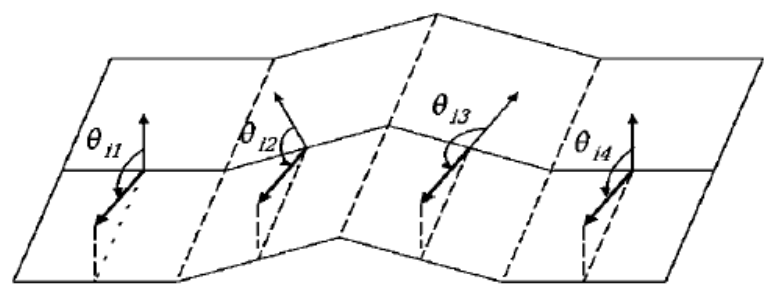

Fig. 5. Incidence Angle when Microwave Radiometer Observes in Perpendicular with Wind Direction.

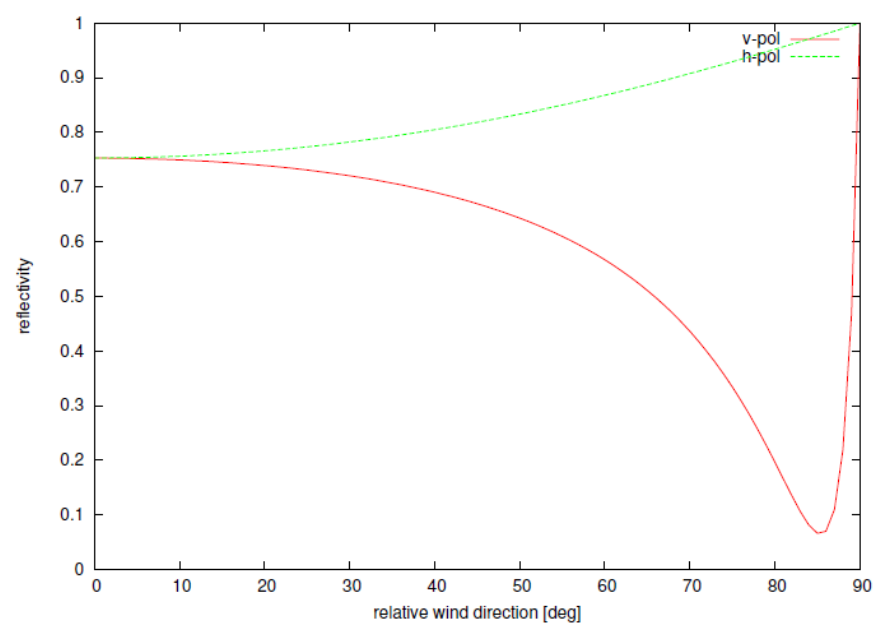

Fig. 6. Relation between Reflectivity and Relative Wind Direction. 
First, in the case of horizontally polarized waves, the incident angle of ASMR-E is 55 deg. In the vicinity, the reflectance increases as the incident angle increases. On the contrary, reflectance becomes greater in accordance with the incident angle becomes smaller.

A similar relationship can be seen at $10 \mathrm{GHz}, 18 \mathrm{GHz}$ and 23GHz. Therefore, RWD $=90$ deg., which increases the average observation angle of incidence. Then, the horizontal polarization component of the upper atmosphere brightness temperature becomes large, and the vertical polarization becomes small. In addition, $\mathrm{RWD} \simeq 0^{\circ}$ and $/$ or $180^{\circ}$, which has a relatively small average observation angle. Then, it can be expected that the horizontal polarization of the upper atmosphere brightness temperature becomes smaller, and the vertical polarization becomes larger. However, since the exact gradient distribution is unknown, it is not known how much the relative wind direction affects the brightness temperature. In this study, the effect was analytically derived.

\section{Proposed Method}

In this study, the effect of the relative wind direction on the brightness temperature was analyzed by comparing the brightness temperature affected by the relative wind direction with the brightness temperature not affected. In this case, the brightness temperature includes not only the downward component of atmospheric radiation, but also the upward component of atmospheric radiation and radiation from the sea surface.

As the brightness temperature affected by the relative wind direction, the brightness temperature data (TB: Brightness Temperature) of Level 1B of the microwave radiometer AMSR-E mounted on the AQUA was used. AMSR-E Leve1 $1 \mathrm{~B}$ products have polarization types of horizontal polarization and vertical polarization, and the center frequencies of the frequency bands are 6.9, 10.7, 18.7, 23.8, 36.5, 89.0GHz, respectively. In this study, we used horizontal and vertical polarization in the frequency band below $23.8 \mathrm{GHz}$ and used winter data that can be expected to have relatively strong winds and summer data that can be expected to have relatively weak winds.

Table I shows the time zones of the observation data. In the table, start time and end time indicate the observation start and end times in GMT, respectively. The observation positions are shown in Fig. 7. ASMR-E data for half a lap is 196 columns $\mathrm{x}$ about 2000 rows. It is the brightness temperature data of about 3920 opening points. Furthermore, the latitude range is relatively strong at latitude 60 north, from latitude 60 south. Therefore, we targeted about 261333 predictable data. Estimates at all points are shown in Table I.

TABLE I. TIME ZONES OF THE OBSERVATION DATA

\begin{tabular}{|l|l|l|l|}
\hline No & Date & Start & End \\
\hline 1 & Aug.11 & $23: 21$ & $00: 11$ \\
\hline 2 & Aug.12 & 11.43 & $12: 33$ \\
\hline 3 & Dec.8 & $23: 27$ & $00: 18$ \\
\hline 4 & Dec.9 & $11: 49$ & $12: 39$ \\
\hline
\end{tabular}

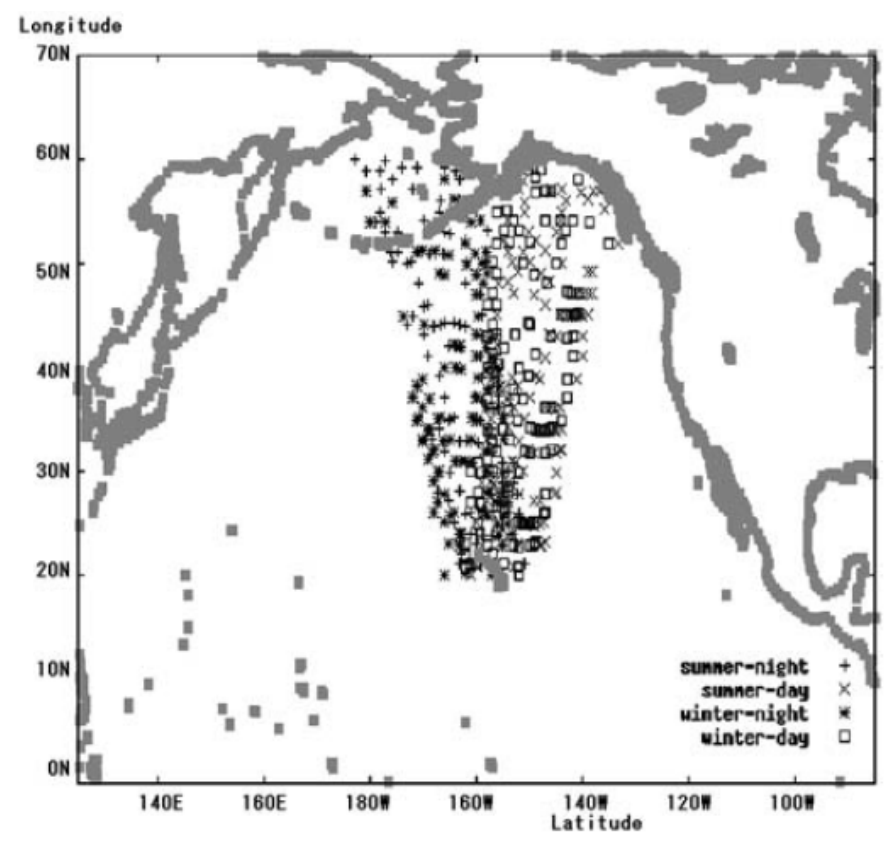

Fig. 7. Observation Positions.

Since it takes too much time, we used one-thousandth of the data and the data for GMT at random sampling. Therefore, the amount of data of the observed positions used is 261 . We prepared representative summer / winter and day / night datasets and used them for the experiment. Wentz's microwave radiation transfer model based on global meteorological data (GTAD83.2: Global Tropospheric Analyses dS083.2) provided by the National Centers for Environmental Prediction (NCEP) as a brightness temperature that is not affected by the wind direction.

The brightness temperature derived using the above was used. The global meteorological data used at this time is the data at the same position and time as the ASMR-E data to be compared. The variables given as the input value of the microwave radiation transmission model are the absolute azimuth of the wind (WD: Wind Direction), the sea surface temperature (SST: Sea Surface Temperature), Precipitable water (PW), cloud water amount (CW: Cloud Liquid water). The output value is represented by the brightness temperature TB of the microwave. All these variables are obtained from GTAD83.2. GTAD83.2 has a mesh size of 1 by 1 degrees and is updated every 6 hours for ASMR-E data and GTAD.

Since the data of GTAD83.2 has a difference in the observation position, the data of GTAD83.2 was linearly interpolated to correspond to the data position of ASMR-E. The relative wind direction was defined by equations (6) to (8).

$\begin{aligned} \theta_{\text {amsr }} & =\left[\begin{array}{l}\cos \left(\theta_{\text {amsr }}\right) \\ \sin \left(\theta_{\text {amsr }}\right)\end{array}\right] \\ \theta_{\text {wind }} & =\left[\begin{array}{l}\cos \left(\theta_{\text {wind }}\right) \\ \sin \left(\theta_{\text {wind }}\right)\end{array}\right] \\ R W D & =\cos ^{-1}\left(\frac{\theta_{\text {amsr }} \theta_{\text {wind }}}{\left|\theta_{\text {amsr }}\right|\left|\theta_{\text {wind }}\right|}\right)\end{aligned}$ 
RWD represents the relative wind direction, $\theta_{\text {amsr }}$ is the ASMR-E observation azimuth, and $\theta_{\text {wind }}$ is the wind azimuth.

The calculation result of the radiation transfer model is used as the data that is not affected by the relative wind direction, and it is defined by the following formula.

$T B_{\text {simu }}=\operatorname{SIMU}(s s t, w s, p w, c w)$

where $T B$ is the brightness temperature that is not affected by the relative wind direction, SIMU() is the microwave radiation transmission model, and the arguments are the sea surface temperature, sea wind speed, precipitable water, and cloud water obtained from GTAD083.2, respectively. Furthermore, the brightness temperature affected by the relative wind direction is represented by $T B_{a m s r}$, and the relative influence of the wind direction is defined by the following formula.

$\Delta T B=T B_{a m s r}-T B_{\text {simu }}$

Furthermore, since it is considered that the influence of the wind direction depends on the wind speed, the $\triangle T B$ call at a certain wind speed is represented by $\triangle T B \mid w s$. This relationship was approximated by Eq. (11).

$\left.\Delta T B\right|_{w s} \cong A R W D^{2}+B R W D+C$

Fig. 8 to 11 show examples of horizontal polarization in the $10 \mathrm{GHz}$ band. Examples of band vertical polarization are shown in Fig. 12 to 13. From Fig. 8 to Fig. 13, it can be seen that the coefficients $a_{1}$ and $c_{3}$ in Eq. (11) change depending on the wind speed. The relationship was approximated by equations (12), (13), and (14).

$$
\begin{aligned}
& A \cong a_{1} W S^{2}+a_{2} W S+a_{3} \\
& B \cong b_{1} W S^{2}+b_{2} W S+b_{3} \\
& C \cong c_{1} W S^{2}+c_{2} W S+c_{3}
\end{aligned}
$$

Equations (11) to (14) and nine approximation coefficients from $a_{1}$ to $c_{3}$ can be used to add the RWD effect to the physical model. As a result, the coefficients in Table II were derived.

Examples of horizontal polarization in the $10 \mathrm{GHz}$ band are shown in Fig. 14, 15, and 16.
From Fig. 8 to Fig. 10, the characteristics of the relative wind direction dependence become clear as the wind speed increases. That is, the observed brightness temperature increases as the relative wind direction approaches 90 degrees. This feature is prominent in horizontal polarization.

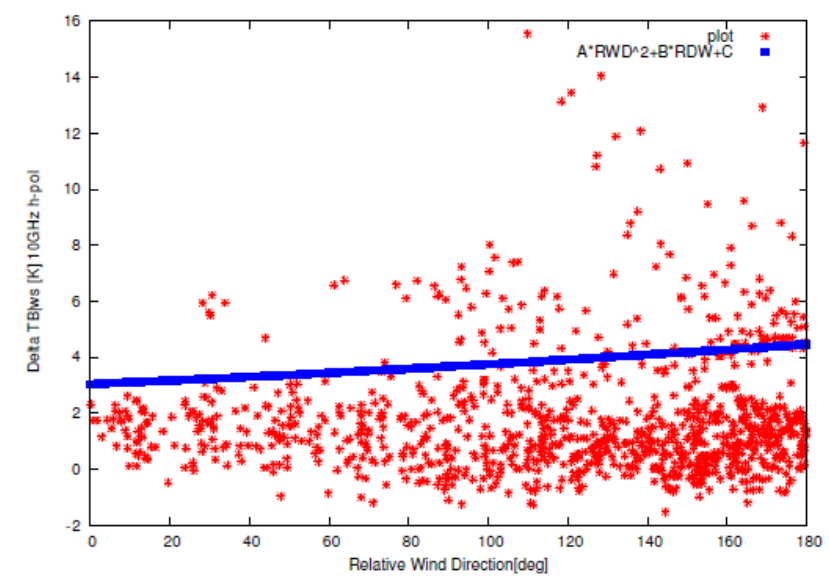

Fig. 8. Relation between Relative Wind Direction and Delta Brightness Temperature (10GHz H-pol $1.26 \mathrm{~m} / \mathrm{s})$.

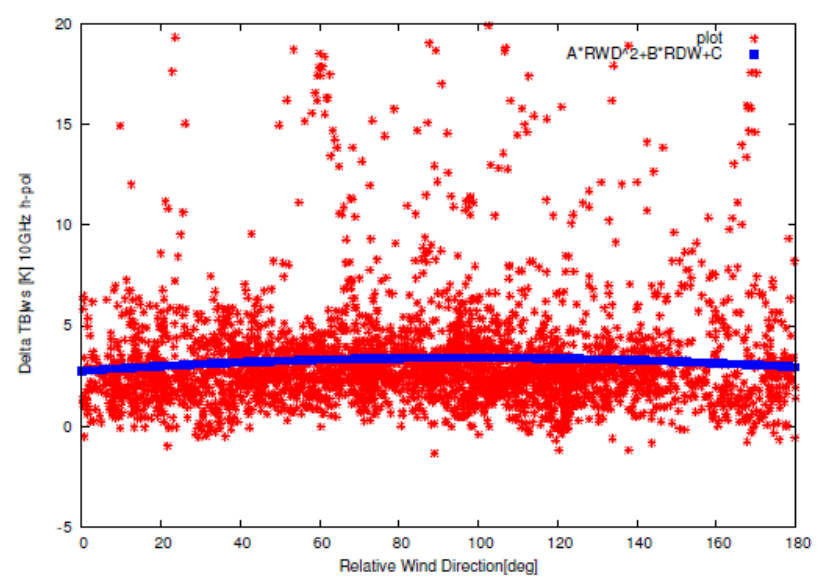

Fig. 9. Relation between Relative Wind Direction and Delta Brightness

\begin{tabular}{|c|c|c|c|c|c|c|c|c|c|c|}
\hline & & a1 & $\mathrm{a} 2$ & a3 & b1 & b2 & b3 & c1 & c2 & c3 \\
\hline \multirow[t]{2}{*}{$6 \mathrm{GHz}$} & V-pol & 0.011 & -0.202 & 0.839 & 0.001 & 0.033 & -0.702 & 0.007 & -0.264 & 3.502 \\
\hline & H-pol & -0.003 & -0.085 & 0.488 & 0.038 & -0.251 & 0.42 & -0.041 & 0.231 & 3.134 \\
\hline \multirow[t]{2}{*}{$10 \mathrm{GHz}$} & V-pol & 0.003 & -0.071 & 0.0327 & -0.002 & 0.033 & -0.462 & 0.011 & -0.253 & 3.518 \\
\hline & H-pol & -0.006 & -0.036 & 0.303 & 0 & 0.448 & -2.22 & -0.027 & 0.115 & 3.26 \\
\hline \multirow[t]{2}{*}{$18 \mathrm{GHz}$} & V-pol & 0.005 & -0.107 & 0.547 & -0.003 & 0.0023 & -0.684 & 0.014 & -0.182 & 4.688 \\
\hline & H-pol & -0.006 & -0.056 & 0.274 & 0.023 & 0.056 & -0.383 & -0.011 & -0.049 & 6.286 \\
\hline \multirow[t]{2}{*}{$23 \mathrm{GHz}$} & V-pol & 0.01 & -0.18 & 0.739 & 0.002 & -0.187 & 0.719 & 0.025 & -0.178 & 4.209 \\
\hline & H-pol & -0.004 & -0.03 & 0.093 & 0.016 & -0.097 & 0.761 & -0.024 & 0.477 & 4.392 \\
\hline
\end{tabular}
Temperature (10GHz H-pol 7 m/s).

TABLE II. COEFFICIENTS FOR EQUATIONS (11) TO (14) 


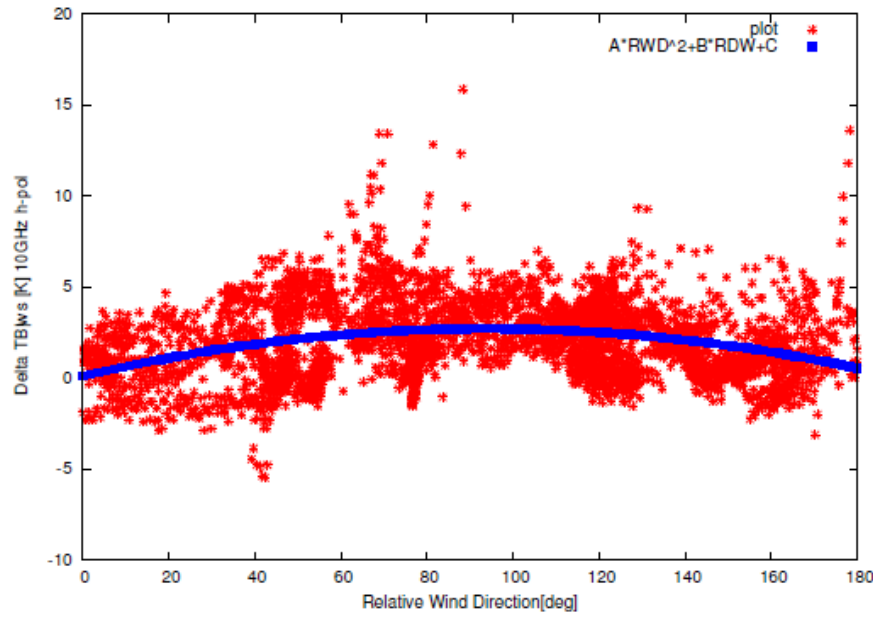

Fig. 10. Relation between Relative Wind Direction and Delta Brightness Temperature (10GHz H-pol 12.93 m/s).

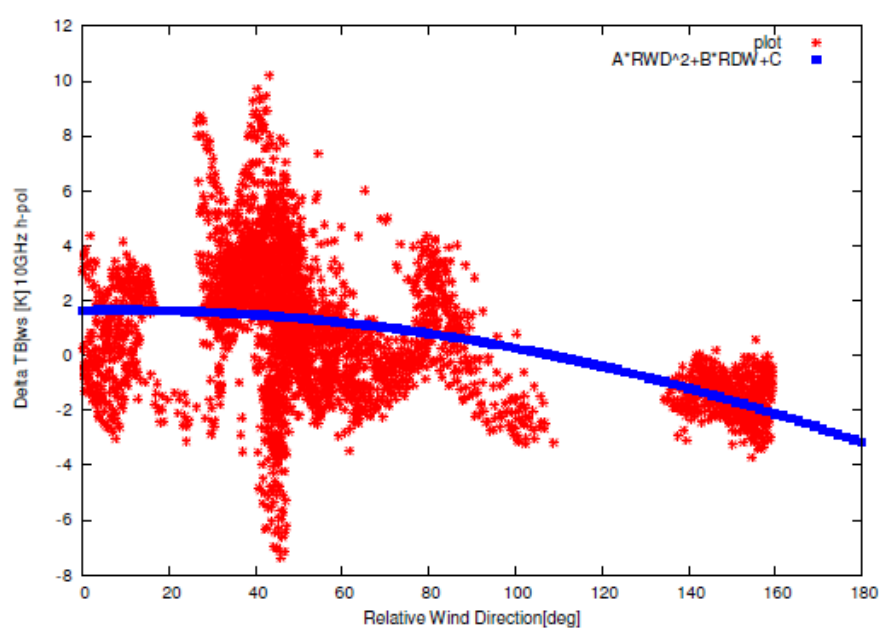

Fig. 11. Relation between Relative Wind Direction and Delta Brightness Temperature (10GHz H-pol 19.09 m/s).

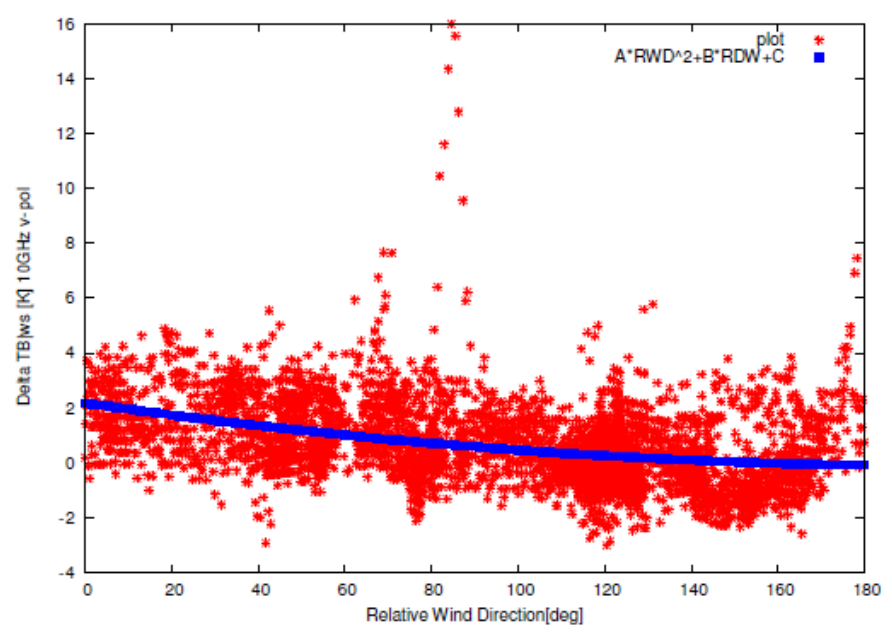

Fig. 12. Relation between Relative Wind Direction and Delta Brightness Temperature (10GHz V-pol 12/93 m/s).

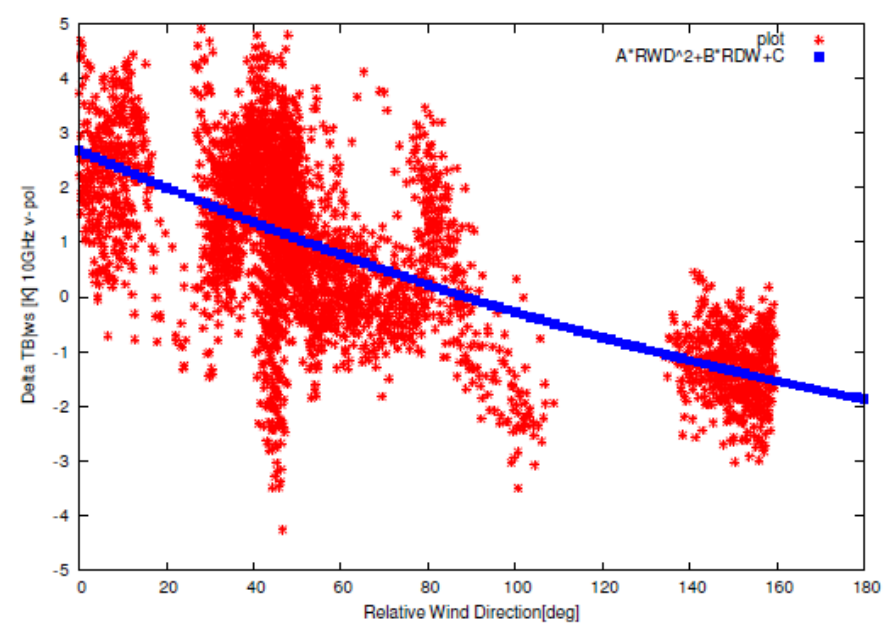

Fig. 13. Relation between Relative Wind Direction and Delta Brightness Temperature (10GHz V-pol $19.09 \mathrm{~m} / \mathrm{s})$.

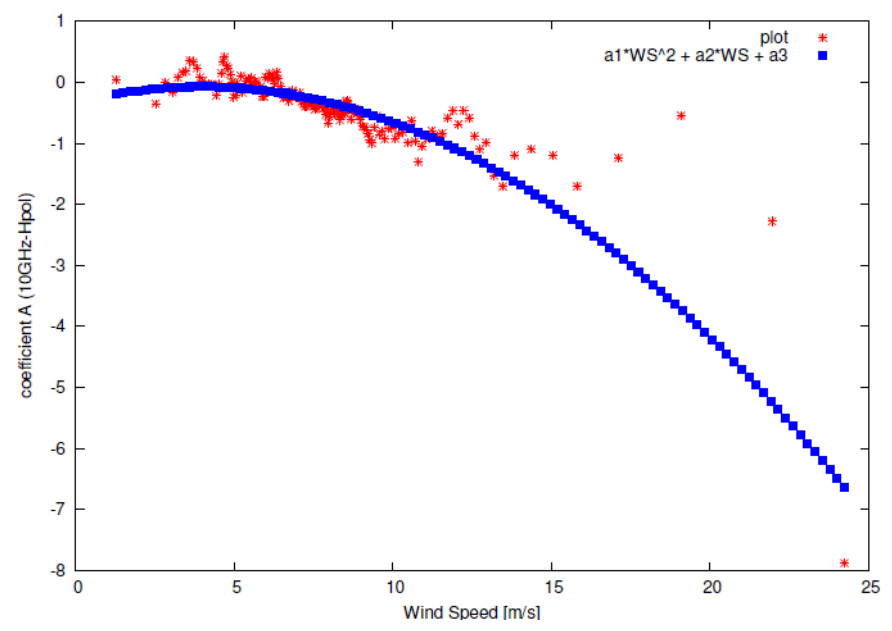

Fig. 14. Relation between Coefficient A and Wind Speed (10GHz H-pol).

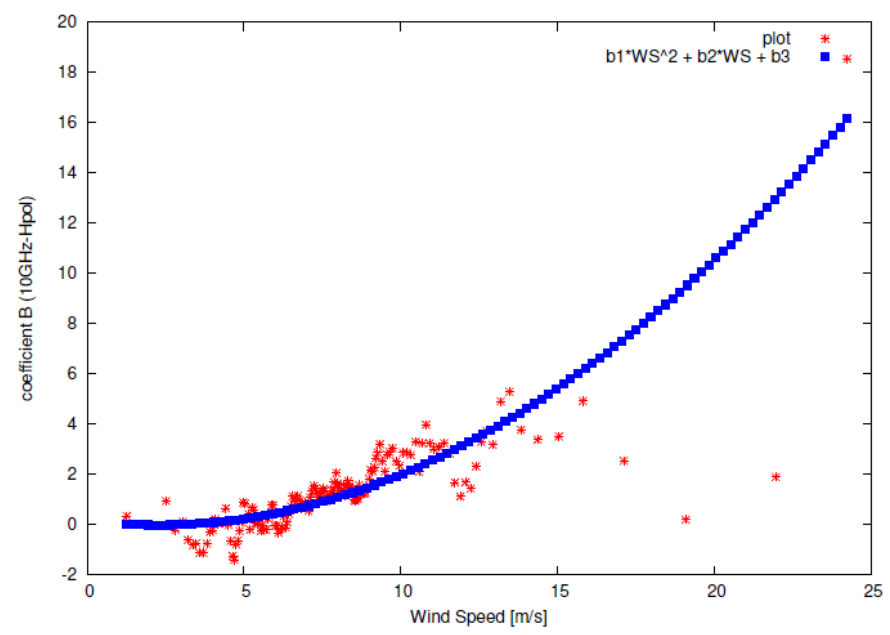

Fig. 15. Relation between Coefficient B and Wind Speed (10GHz H-pol). 


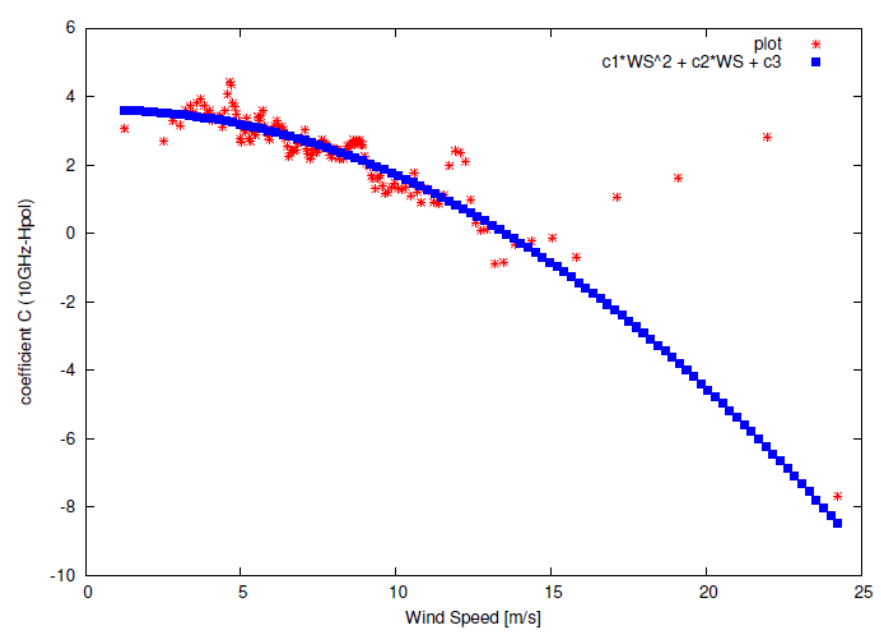

Fig. 16. Relation between Coefficient C and Wind Speed (10GHz H-pol).

As shown in Fig. 14 to Fig. 16, the change in $\triangle T B$ when the wind speed exceeds about $15 \mathrm{~m} / \mathrm{s}$ is clearly different from that when the wind speed exceeds about $15 \mathrm{~m} / \mathrm{s}$. It is known that wind waves grow when the wind speed increases and collapse when the wind speed exceeds a certain level. From this, it is considered that the feature of about $15 \mathrm{~m} / \mathrm{s}$ is due to the collapsing waves. Another possible cause is the white waves that occur when the wind speed is strong. In addition, Fig. 12 and Fig. 14 clearly show the difference of $\triangle T B$ when observed from the front and the rear of the collapsing wave.

When the RWD is 90 degrees, that is, when observing from behind the collapsing wave, the observed $T B$ becomes large; when RWD> 90 degrees, that is, when observing from the front of the collapsing wave, the observation becomes small.

\section{EXPERIMENT}

Sea wind direction, wind speed, sea surface temperature, precipitable water, cloud water estimations are conducted. Using Equations (11) to (14) and Table II described in the previous chapter, the effect of the relative wind direction on the brightness temperature can be understood. Therefore, by adding the brightness temperature derived using this equation and the coefficient to the microwave radiation transfer equation of Wentz, the radiation transfer equation considering the relative wind direction can be obtained.

This function is non-linear. Therefore, to determine the physical quantity at sea from the brightness temperature at the upper end of the atmosphere, the inverse problem must be solved. To solve this reverse problem, we used Simulated Annealing, which was proposed by Arai. The luminance temperature used for the estimation was ASMR-E Level 1B (horizontal and vertical polarization 6,10,18,23 $\mathrm{GHz}$ band), which was the same as the data used in the previous chapter. The estimated physical quantities are sea surface temperature SST, sea surface temperature WS, relative wind direction RWD, precipitable water PW, and cloud water volume CW, and the estimated location is over the Pacific Ocean (20-60 north latitude, 180-230 east longitude).
The correct answer data used to compare the estimation results is the global meteorological data GTAD83.2 provided by UCEP, which is also the same as the one used in the previous chapter. As in the previous chapter, linear interpolation was performed to positionally correspond to the ASMR-E data. The standard deviation between the value estimated from the luminance temperature of AMSR-E and the correct answer value obtained from GTAD83.2 was used as the standard deviation. The standard difference was derived by Equation 15.

$$
A c u=\sqrt{\frac{\sum_{i=1}^{n}\left(A n s_{i}-X_{i}\right)^{2}}{n}}
$$

where $A c u$ is the estimation accuracy, $A n s_{i}$ is the correct value obtained from GTAD83.2, $X_{i}$ is the estimated physical quantity, $\mathrm{n}$ is the data number, and $\mathrm{d}$ is the total number of estimated data. In addition, when calculating this estimation accuracy, the estimation point where the error (energy $E$ ) due to simulated annealing was clearly large was rejected because the estimation result is clearly wrong. Table III summarizes the estimation accuracy of each estimated physical quantity. In addition, Table III also summarizes the estimation accuracy when estimating with the conventional model as a target for comparison. The data numbers are shown in Table I.

The conventional model is a method using Wentz's microwave radiation transfer equation and improved simulated annealing by Arai and Sakakibara. Hereafter, the algorithm when considering the influence of the relative wind direction derived in this study is called the modified model and is distinguished from the conventional model. The modified model is Wentz's microwave radiation transfer equation modified by the influence of the relative wind direction shown in the previous chapter. Regarding the wind speed, the estimation accuracy improved except for data 1 . The reason why the estimation accuracy of data 1 deteriorated is that it is estimated at the same time.

It is probable that the estimation accuracy of the relative wind direction was poor. On the contrary, the data 3 in which the estimation accuracy of the relative wind direction is greatly improved also greatly improves the estimation accuracy of the wind speed. It can be seen that to improve the accuracy of wind speed estimation in consideration of the relative wind direction, it is necessary to improve the estimation accuracy of the relative wind direction. However, the effect of the relative wind direction on the observed luminance temperature is small compared to other physical quantities estimated at the same time. Therefore, in the estimation method used in this study, it is considered that the estimation accuracy of the relative wind direction deteriorated due to the error that occurred when calculating other physical quantities.

Compared to data 1 and data 2 observed in summer, the estimation accuracy of wind speeds in data 3 and data 4 observed in winter has improved significantly. This is thought to be because the relative wind direction has a stronger effect in winter when the wind is strong than in summer when the wind is weak. 
TABLE III. SUMMARY OF THE ESTIMATION ACCURACY WHEN ESTIMATING WITH THE CONVENTIONAL MODEL AS A TARGET FOR COMPARISON

\begin{tabular}{|l|l|l|l|l|l|l|}
\hline Model & $\begin{array}{l}\text { No } \\
\cdot\end{array}$ & $\begin{array}{l}\text { SST[K } \\
]\end{array}$ & $\begin{array}{l}\text { WS[m/s } \\
]\end{array}$ & $\begin{array}{l}\text { RWD[deg } \\
]\end{array}$ & $\begin{array}{l}\text { PW[kg/m² } \\
]\end{array}$ & $\begin{array}{l}\text { CW[kg/m2 } \\
]\end{array}$ \\
\hline \multirow{4}{*}{ Existing } & 1 & 2.63 & 2.13 & 86.92 & 2.45 & 0.004 \\
\cline { 2 - 7 } & 2 & 1.26 & 2.39 & 67.69 & 1.28 & 0.08 \\
\cline { 2 - 7 } & 3 & 1.53 & 2.01 & 62.65 & 2.92 & 0.08 \\
\cline { 2 - 7 } & 4 & 1.32 & 2.52 & 52.64 & 2.75 & 0.07 \\
\hline \multirow{4}{*}{$\begin{array}{l}\text { Propose } \\
\text { d }\end{array}$} & 1 & 0.96 & 2.15 & 99.76 & 2.25 & 0.04 \\
\cline { 2 - 7 } & 2 & 1.19 & 1.91 & 56.53 & 1.83 & 0.1 \\
\cline { 2 - 7 } & 3 & 1.71 & 0.78 & 18.12 & 1.3 & 0.01 \\
\cline { 2 - 7 } & 4 & 1.37 & 1.76 & 68.02 & 1.29 & 0.05 \\
\hline
\end{tabular}

Since the estimation method used in this study is a method of estimating physical quantities at the same time, the estimation accuracy of other physical quantities is affected as well as the change in the estimation accuracy of the wind speed, as shown in Tab1e III. The accuracy of sea surface temperature estimation has improved in summer, and the accuracy of precipitable water estimation has improved in winter.

\section{CONCLUSION}

By comparing the observed brightness temperature affected by the relative wind direction with the observed brightness temperature not affected, the effect of the relative wind direction on the observed brightness temperature was found. In particular, we were able to extract clear features in horizontally polarized waves. In addition, these features could be expressed by equations and coefficients. In the case of the data used in this study, it was found that the estimation accuracy of the wind speed changed by using the model in which the proposed relative winds direction affects the luminance temperature.

When the estimation accuracy of the relative wind direction estimated at the same time was relatively good, the estimation accuracy of the wind speed was also improved. However, if the estimation accuracy of the relative wind direction estimated at the same time is poor, the estimation accuracy of the wind speed may also deteriorate. Therefore, it was also found that it is necessary to improve the estimation accuracy of the relative wind direction in order to improve the estimation accuracy of the wind speed by the proposed model. In addition, the proposed model is a symmetric function with the observed brightness temperature deviation as the peak when the relative wind direction is 90 degrees, and it is considered that the deviation becomes smaller when the relative wind direction is smaller or larger than this.

Considering that the quadratic function approximation is the first-order approximation, the relationship between the relative wind direction and the observed brightness temperature was approximated by a quadratic equation. However, it is necessary to consider a more appropriate approximation function in consideration of physical grounds.
In this study, the effect of the relative wind direction on the luminance temperature was attributed to changes in reflectance. Especially when the wind speed exceeds about 15 $\mathrm{m}$, the optimum approximation formula can be obtained by understanding the physical phenomena related to the relative wind direction, such as the change in brightness temperature due to the collapsing waves that occur when the wind speed is strong, it is conceivable then.

In this study, the proposed method was evaluated by comparing it with the conventional method using NCEP GDAS as the correct answer, but the errors included in NCEP GDAS will be examined in the future. The error analysis and sensitivity solution in the simultaneous estimation of multiple physical parameters and the simultaneous estimation for improving the accuracy of wind direction determination are left to the references.

\section{FUTURE RESEARCH WORK}

In this paper, we have shown a method for classifying each pixel for a certain image, but the proposed method can be applied not only to images but also to various sets with values for each element, such as remote sensing, GIS, and it is considered that it can be widely applied to other data mining. Further research works are required for the other applications not only rice paddy field detection with SAR imagery data.

\section{ACKNOWLEDGMENT}

The authors have received regular discussions, useful opinions and suggestions. Remote Sensing Systems Co., Ltd., Dr. Frank J. Wentz, Professor Masanori Neda of Kyoto University, Professor Naoto Ebuchi of Hokkaido University, JAXA Akira Shibata I am deeply grateful to the doctor. In addition, the master's degree of our graduate school that cooperated with the data collection experiment and analysis.

We would like to thank Mr. Atsushi Sakakibara of former student of Saga University and Prof. Dr. Hiroshi Okumura and Prof. Dr. Osamu Fukuda of Saga University for his valuable comments and suggestions.

\section{REFERENCES}

[1] Kohei Arai: Basic Theory of Remote Sensing, Academic Book Publisher, 2001.

[2] Kohei Arai: Self-study Remote Sensing, Morikita Publishing, 2004.

[3] Japan Aerospace Exploration Agency:-Handbook for Utilizing Earth Observation Data - AMSR-E Flat --- ”, 2006. http://www.eorc.jaxa.jp/hatoyama/amsr-e/amsr-e handbook "(6Jun. 2007).

[4] Frank Wentz: ASMR Ocean Algorithm, Remote Sensing System, 2002.

[5] Akira Shibata: Observation of atmosphere, ocean, and land with multiwavelength microwave radiometer, Journal of Japan Remote Sensing Society Special Feature: Radio Waves and Remote Sensing, 12,1,59-64, 1992.

[6] Ara1, K.and J.Sakakibara: Estimation 0fSST, wmd speed and water vapor W1th microwave radiometer data based on simulated anealing, Advances III Space Research, 37,12, 2202-2207, 2006.

[7] M. Konda, A.Shibata, N.Ebuchi and K.Arai: Correction of the effect of relative wind directionon wind by AMSR, Journal of Oceanography, 64,395-404, 2006.

[8] Dennies J. Shea: "An Introduction to Atmospheric and Oceanographic Data", 1994. http://dss.ucar.edu/docs/data-intro-technote/tn-404.pdf 
[9] M.Matsumoto and Kohei Arai, Simplified expression of the radiative transfer equation in thermal infrared windiow spectrum, Proc.of the IGARSS'93, 673-675, 1993.

[10] N.Ebuchi, Kohei Arai, et.al., Evaluation of vector winds observed by NSCAT in the seas around Japan, Journal of Ocean Society of Japan, Vol.56, No.5, pp.495-505,(2000).

[11] Kohei Arai, Polarization sensitivity of the ocean surface together with wind vector derived from POLDER and NSCAT on ADEOS, Proceedings of the NASA Oceanography Scientific Conference, Florida, USA, 2001.

[12] Kohei Arai and Jun Sakakibara, Estimation of SST, wind speed and water vapor with microwave radiometer data based on simulated annealing, Abstracts of the 35th Congress of the Committee on Space Research of the ICSU, A1.1-0130-04, (2004).

[13] Kohei Arai and Jun Sakakibara, Simultaneous estimation of sea surface temperature, wind speed and water vapor with AMSR-E data based on improved simukated anneiling, Proceedings of the Renewable Energy Resources Symposium, 00547, 2006.

[14] Kohei Arai, Space and time retrieval of tide wind speed and wave height with altimeters onboard satellites based on PostGIS system, Proceedings of the Renewable Energy Resources Symposium, 00548, 2006.

[15] Kohei Arai and J.Sakakibara, Estimation of SST, wind speed and water vapor with microwave radiometer data based on simulated annealing, Advances in Space Research, 37, 12, 2202-2207, 2006.

[16] M.Konda, A.Shibata, N.Ebuchi and Kohei Arai, Correction of the effect of relative wind direction on wind speed derived by AMSR, Journal of Oceanography, 64, 395-404, 2006.

[17] Kohei Arai, Data fusion between microwave and thermal infrared radiometer data and its application to skin sea surface temperature, wind speed and salinity retrievals, International Journal of Advanced Computer Science and Applications, 4, 2, 239-244, 2013.

[18] Kohei Arai, Comparative Study of optimization Methods for Estimation of Sea Surface Temperature and Ocean Wind wit microwave Radiometer data, International Journal of Advanced Research on Artificial Intelligence, 5, 1, 1-6, 2016.

\section{AUTHOR's PROFILE}

Kohei Arai, He received BS, MS and PhD degrees in 1972, 1974 and 1982, respectively. He was with The Institute for Industrial Science and Technology of the University of Tokyo from April 1974 to December 1978 also was with National Space Development Agency of Japan from January, 1979 to March, 1990. During from 1985 to 1987, he was with Canada Centre for Remote Sensing as a Post Doctoral Fellow of National Science and Engineering Research Council of Canada. He moved to Saga University as a Professor in Department of Information Science on April 1990. He was a councilor for the Aeronautics and Space related to the Technology Committee of the Ministry of Science and Technology during from 1998 to 2000. He was a councilor of Saga University for 2002 and 2003. He also was an executive councilor for the Remote Sensing Society of Japan for 2003 to 2005. He is a Science Council of Japan Special Member since 2012. He is an Adjunct Professor of University of Arizona, USA since 1998. He also is Vice Chairman of the Science Commission "A" of ICSU/COSPAR since 2008 then he is now award committee member of ICSU/COSPAR. He wrote 55 books and published 620 journal papers as well as 450 conference papers. He received 66 of awards including ICSU/COSPAR Vikram Sarabhai Medal in 2016, and Science award of Ministry of Mister of Education of Japan in 2015. $\mathrm{He}$ is now Editor-in-Chief of IJACSA and IJISA. http://teagis.ip.is.sagau.ac.jp/index.html 